\title{
LIPÍDIOS PLASMÁTICOS APÓS ESPLENECTOMIA TOTAL E PARCIAL EM CÃES
}

\section{THE PLASMA LIPIDS AFTER TOTAL AND PARTIAL SPLENECTOMY IN DOGS}

\author{
Danilo Nagib Salomão Paulo, TCBC-ES \\ Alcino Lázaro da Silva, TCBC-MG ${ }^{2}$
}

\begin{abstract}
RESUMO: Objetivo: Avaliar o efeito da esplenectomia total e parcial (hemiesplenectomia e esplenectomia subtotal) no lipidograma de cães. Método: Foram operados 38 animais, adultos, machos, mestiços, com peso entre $13 \mathrm{~kg}$ e $15 \mathrm{~kg}$. No pré-operatório, depois do exame clínico e jejum de 12 horas de uma dieta-padrão, foram realizados hemograma e lipidograma. Após anestesia geral com tiopental sódico os cães foram submetidos à laparotomia mediana supra e infra-umbilical. Procedeu-se no grupo 1, de sete cães, apenas à manipulação do baço (simulado); no grupo 2, de nove cães, à hemiesplenectomia cranial; no grupo 3, de nove cães, à esplenectomia subtotal, com preservação do pólo inferior do baço, após ligadura e secção dos troncos principais dos vasos esplênicos próximos ao hilo; e no grupo 4, de 13 cães, à esplenectomia total. Resultados: No grupo 4 (esplenectomia total), quando comparamos os valores do lipidograma dos diferentes períodos pós-operatórios com os do pré-operatório, os nossos resultados mostraram que houve aumento significante do colesterol total em todos os períodos pós-operatórios ( $\mathrm{p}<0,05, \mathrm{p}<0,01)$. O HDL aumentou significantemente no $7^{\mathrm{o}}(\mathrm{p}<0,01)$ e $28^{\circ}$ dias de pós-operatório (PO) $(\mathrm{p}<0,05)$, e não significantemente $(\mathrm{p}>0,05)$ no 56을 PO O LDL aumentou significantemente no $56^{\mathrm{o}} \mathrm{PO}(\mathrm{p}<0,01)$ em relação também aos demais períodos do pós-operatório. Os triglicerídeos e VLDL não apresentaram alterações significantes. Nos animais dos grupos 1 e 3 não houve alterações significantes. Nos do grupo 2, houve aumento significante do colesterol no $7^{\circ}$ PO. Não houve diferença estatisticamente significante de peso entre os remanescentes das esplenectomias parciais. Conclusões: A análise dos resultados nos permitiu concluir que a esplenectomia total induz aumento significante do colesterol total, das frações HDL e LDL, enquanto os níveis de triglicerídios e a fração VLDL não sofrem alterações; a conservação da metade inferior do baço ou a esplenectomia subtotal, com preservação do pólo inferior, protege o animal de alterações lipídicas significantes.
\end{abstract}

Descritores: Esplenectomia parcial; Esplenectomia subtotal; Esplenectomia e colesterol.

\section{INTRODUÇÃO}

Os efeitos da esplenectomia no metabolismo lipídico têm sido relatados em trabalhos clínicos e experimentais ${ }^{1-7}$.

Pacientes com doenças mieloproliferativas (policitemia vera, mielofibrose, metaplasia mielóide) associadas à esplenomegalia apresentam diminuição dos valores do co- lesterol total, HDL, apolipoproteína B e apolipoproteína A1. Esses valores normalizam-se após as ressecções ${ }^{1}$. Os autores admitem que o baço é um importante local para o catabolismo do LDL. Tem sido relatado que a ablação do baço induz aumento do colesterol total em pacientes com hiperesplenismo (esferocitose hereditária, leucemia mielóide crônica e policitemia vera). Na esferocitose hereditá-

1. Professor Titular de Técnica Operatória e Cirurgia Experimental da Escola Superior de Ciências da Santa Casa de Misericórdia de Vitória-ES.

2. Professor Titular de Cirurgia do Aparelho Digestivo da Faculdade de Medicina da UFMG.

Recebido em 11/12/2000

Aceito para publicação em 10/5/2001

Trabalho realizado no Laboratório da Disciplina de Técnica Operatória e Cirurgia Experimental da Escola Superior de Ciências da Santa Casa de Misericórdia de Vitória-ES. 
ria os lipídios plasmáticos estão diminuídos. Após a exérese esplênica, os lipídios são parcialmente restaurados². As alterações lipídicas que ocorrem após esplenectomia por trauma poderiam explicar em parte a alta incidência de infarto do miocárdio observada em veteranos que estiveram na Segunda Guerra Mundial ${ }^{3}$.

$\mathrm{King}^{4}$, em 1914, encontrou aumento do colesterol em cães, após a remoção esplênica. Ham e Furneaux ${ }^{5}$, em 1969, não constataram alterações na lipase lipoprotéica nem nos lipídios plasmáticos duas semanas após a esplenectomia em cães. Asai et al. ${ }^{6}$ observaram que coelhos, que se alimentavam de produtos com altos níveis de colesterol, apresentaram aumento significante de colesterol, triglicerídeos, fosfolipídios, e baixos níveis de HDL, após retirada do baço, em relação ao grupo simulado. Foi também descrito aumento significativo dos triglicerídeos e diminuição do HDL em ratos esplenectomizados alimentados normalmente em relação ao grupo simulado. Esse fato foi também detectado em ratos com alimentação enriquecida com colesterol ${ }^{7}$.

Esses trabalhos clínicos e experimentais falam a favor de uma interferência do baço no metabolismo lipídico. Em cães, conforme relatamos, há uma certa controvérsia sobre a influência desse órgão no metabolismo lipídico. Com base nesses fatos, passamos a indagar se, nesses animais, o baço interfere no metabolismo lipídico e se uma porção desse órgão seria capaz de conservar a função lipídica. Por esse motivo, realizamos um trabalho com os seguintes objetivos: verificar se o baço interfere no metabolismo lipídico de cães, e se a esplenectomia parcial (hemiesplenectomia, esplenectomia subtotal) foi capaz de conservar a referida função.

\section{MÉTODO}

Foram utilizados 38 cães mestiços, machos, com peso entre $13 \mathrm{~kg}$ e $15 \mathrm{~kg}$, alimentados de uma dieta normal (Lord).

Foram submetidos a exame clínico, hemograma e lipidograma no pré-operatório. O lipidograma foi feito no aparelho Vitalab Selectra da Merck. No 7ํㅡ, 28o e 56으 dias pós-operatórios, repetimos os referidos exames.

Os animais foram divididos em quatro grupos:

- Grupo 1- constituido por sete cães, operação simulada (controle);

- Grupo 2 - por nove cães, hemiesplenectomia;

- Grupo 3 - por nove cães, esplenectomia subtotal com preservação do pólo inferior do baço;

- Grupo 4 - por 13 cães, esplenectomia total.

Os animais foram inicialmente sedados com clorpromazina (25mg intramuscular). Em seguida, foi puncionada uma veia da pata dianteira para administração de solução glicosada a $5 \%$. A cefalotina, na dose de $1 \mathrm{~g}$ IV, foi administrada em caráter preventivo. A seguir, foi aplicada uma dose (10mg/kg de peso) de tiopental sódico, e os cães foram intubados sob laringoscopia direta.

A laparotomia foi mediana supra e infra-umbilical com cerca de $12 \mathrm{~cm}$ de comprimento. Nos animais do grupo 1 (controle), fez-se o inventário da cavidade abdominal e a manipulação do baço. Nos animais do grupo 2, reali- zou-se a hemiesplenectomia com preservação da metade inferior. No grupo 3, a esplenectomia subtotal com preservação do pólo inferior, conforme técnica já descrita ${ }^{8}$. No grupo 4, realizou-se a esplenectomia total. $\mathrm{O}$ baço retirado foi medido, pesado e examinado macroscopicamente. A parede abdominal foi suturada por planos. No pós-operatório, os cães receberam dieta idêntica à do pré-operatório, analgésicos (dipirona-2ml intramuscular) e cuidados com a ferida operatória.

Nos 7ำ, 28a e 56으 dias pós-operatórios, foram repetidos o hemograma e o lipidograma. Foram dosados o colesterol total (HDL, LDL e VLDL) e triglicerídeos. Os animais foram mortos para inventário abdominal e retirada do baço, porventura existente, que foi medido e pesado.

Os valores das médias aritméticas (MA) e seus desvios-padrão (DP) correspondentes ao lipidograma do préoperatório foram comparados com os valores dos diferentes períodos pós-operatórios (7o, 28o e 56o). Para isso, o teste estatístico utilizado foi a análise de variância em uma via para medidas repetidas. Sempre que o resultado se mostrou significante, fez-se um tratamento post-hoc, por meio do Tukey's protected T-test visando localizar as diferenças entre os pares de médias abrangidas pela análise. Foi também utilizado "o teste $t$ de Student" para comparar os pesos (MA e DP) do remanescente esplênico da hemiesplenectomia com aquele da esplenectomia subtotal, para inferir a influência do referido remanescente nos valores do lipidograma no pós-operatório. Fixou-se em 0,05 ou $5 \%$ o nível de rejeição da hipótese de nulidade. Assinalamos com um $*(\mathrm{p}<0,05)$ ou dois $* *$ asteriscos $(\mathrm{p}<0,01)$ os valores significantes. Todos os tratamentos estatísticos foram realizados por meio do programa "GB-Stat" versão 40 da "Dynamic Microsystems, Inc" (Maryland, USA) registro no 84009075 .

\section{RESULTADOS}

Foram registrados seis óbitos no pós-operatório. A causa foi evisceração em três, hemorragia intraperitoneal em um cão, desnutrição em um e causa desconhecida em outro.

O aspecto macroscópico do baço foi normal nos grupos 1 e 4 . Nos grupos da hemiesplenectomia e da esplenectomia subtotal, o baço remanescente estava aderido freqüentemente ao omento. Algumas vezes, as aderências se faziam com as alças intestinais ou com a parede abdominal. O peso médio e o desvio padrão (DP) nas ressecções totais foram $167 \pm 74,11 \mathrm{~g}$. O peso médio e o DP dos segmentos de baço retirados no grupo da hemiesplenectomia foram $89,12 \pm 51,41 \mathrm{~g}$. Nesse grupo o peso médio e o DP dos segmentos esplênicos que ficaram foram 66,12 $\pm 29,56$ g. Isso significa que no grupo da hemiesplenectomia, a porção remanescente representou cerca de $42 \%$ do órgão total. $O$ peso médio dos segmentos retirados na esplenectomia subtotal foi $128,87 \mathrm{com}$ DP de 85,31 . O peso médio dos segmentos de baço que restaram e foram retirados por ocasião do óbito foi 59,87 , com DP de $\pm 43,57$. Isso significa que, na esplenectomia subtotal, foram deixados cerca de $31 \%$ do órgão. A análise estatística mos- 
trou que não houve diferença $(\mathrm{p}>0,05)$ entre os pesos (MA e DP) dos baços residuais da esplenectomia subtotal e hemiesplenectomia,

Os resultados do lipidograma podem ser observados nas Tabelas 1, 2, 3, 4 e 5.

Na Tabela 1 podemos observar os seguintes aspectos:

1. Os valores médios e os DP do colesterol, no grupo simulação, não sofreram variações significantes entre o pré-operatório e os diferentes períodos operatórios nem entre os diferentes períodos pós-operatórios.

2. No grupo 2 (hemiesplenectomia), houve aumento dos valores médios e dos DP dos níveis de colesterol no sétimo dia de pós-operatório (PO) em relação ao préoperatório $(\mathrm{p}<0,01)$. Não houve diferença entre os valores de pré-operatório e os dos outros períodos de pósoperatório (28ㅇ e 56으).

3. No grupo 3 (esplenectomia subtotal), não houve diferença entre os valores do lipidograma do pré-operatório e os valores dos diferentes períodos operatórios. Isso também ocorreu entre os diferentes períodos operatórios (Tabelas 1 a 5).

4. No grupo 4 (esplenectomia total) houve aumento $(\mathrm{p}<0,05)$ dos valores médios e dos DP do colesterol entre o préoperatório e os demais periodos pós-operatórios. A diferença só não foi significante entre o sétimo PO e o $28^{\mathrm{o}} \mathrm{PO}(\mathrm{p}>0,05)$.
Na Tabela 2, observamos que não houve diferença estatisticamente significante dos valores médios e dos DP dos triglicerídeos nos diferentes grupos $(\mathrm{p}=0,68$, no grupo simulado; $\mathrm{p}=0,68$, no grupo hemiesplenectomia e $\mathrm{p}=0,51$ no grupo esplenectomia total), nos diferentes períodos operatórios.

Na Tabela 3, observamos que o HDL, no grupo 1 (simulado) e no grupo 2, não sofreu modificação estatisticamente significante nos diferentes períodos operatórios ( $\mathrm{p}=0,41$, grupo simulado; $\mathrm{p}=0,55$ no grupo da hemiesplenectomia). No grupo 3 não houve diferença, embora os níveis de HDL do 7o PO fossem maiores que os níveis do pré-operatório. No grupo 4 é importante observar que o HDL aumentou no 7o $(\mathrm{p}<0,01)$ e $28^{\circ} \mathrm{PO}(\mathrm{p}<0,05)$ em relação ao pré-operatório e não aumentou no $56^{\circ} \mathrm{PO}$.

Na Tabela 4, verificamos os seguintes aspectos: 1) No grupo da operação simulada, não houve diferença entre a MA e os DP do LDL nos diversos períodos operatórios $(\mathrm{p}=0,25) ; 2)$ no grupo 2 , esse fato repetiu-se $(\mathrm{p}=0,50)$; 3 ) no grupo 3 , é importante destacar que os valores médios e o DP do LDL aumentaram no 56ㅇ PO em relação ao pré-operatório e ao 7o e $28^{\circ} \mathrm{PO}(\mathrm{p}<0,01)$. Entre o pré-operatório e o 7ำ e o 28우 PO não houve diferença.

$\mathrm{Na}$ tabela 5 verificamos que não houve diferença estatisticamente significante entre a MA e o DP do VLDL nos diferentes grupos (grupo $1, \mathrm{p}=0,47$; grupo $2, \mathrm{p}=0,52$, grupo $3, \mathrm{p}=0,68$ ) e períodos operatórios.

Tabela 1

Colesterol nos diferentes grupos e períodos operatórios

\begin{tabular}{|c|c|c|c|c|c|c|c|c|c|c|c|c|c|}
\hline & $P R E-O P$ & \multicolumn{2}{|c|}{$7^{\circ} \mathrm{PO}$} & \multirow[b]{2}{*}{$p$} & \multicolumn{2}{|c|}{$28^{o} P O$} & \multirow[b]{2}{*}{$p$} & \multirow[b]{2}{*}{$p^{\prime}$} & \multicolumn{2}{|c|}{$56^{\underline{o}} P O$} & \multirow[b]{2}{*}{$p$} & \multirow[b]{2}{*}{$p^{\prime}$} & \multirow[b]{2}{*}{$p "$} \\
\hline & $M A \quad D P$ & $M A$ & $D P$ & & $M A$ & $D P$ & & & $M A$ & $D P$ & & & \\
\hline $\begin{array}{l}\text { Grupo } 1 \\
\text { (simulado) } \\
7 \text { casos }\end{array}$ & $128,28 \pm 17,15$ & 147,14 & $\pm 22,10$ & NS & 151 & $\pm 28,80$ & NS & NS & 160,16 & $\pm 46,40$ & NS & NS & NS \\
\hline $\begin{array}{l}\text { Grupo } 2 \\
\text { (hemiesple- } \\
\text { nectomia) } \\
9 \text { casos }\end{array}$ & $130,22 \pm 28,36$ & 173,44 & $\pm 67,41$ & $* *$ & 144,4 & $\pm 15,60$ & NS & NS & 142,57 & $\pm 15,12$ & NS & NS & NS \\
\hline $\begin{array}{l}\text { Grupo } 3 \\
\text { (esplenectomia } \\
\text { subtotal) } \\
9 \text { casos }\end{array}$ & $150,22 \pm 44,24$ & 177,33 & $\pm 46,97$ & NS & 157,77 & $\pm 59,32$ & NS & NS & 156,44 & $\pm 53,11$ & NS & NS & NS \\
\hline $\begin{array}{l}\text { Grupo } 4 \\
\text { (esplenectomia } \\
\text { total) } \\
13 \text { casos }\end{array}$ & $113,30 \pm 17,16$ & 152 & $\pm 20,34$ & $*$ & 151,8 & $\pm 51,57$ & $*$ & NS & 200,66 & $\pm 91,25$ & $* *$ & $*$ & $*$ \\
\hline
\end{tabular}

Análise de variância de medidas repetidas. Protected T test. $p$ - $^{*} \rightarrow$ significante quando for menor que 0,05. $p * * \rightarrow$ significante quando for menor que 0,01 p p-em relação ao pré-op; p'- em relação ao 7o P.O; p” em relação ao 28 o. NS- não significante. MA- Média aritmética DP-Desvio-padrão. PRE-OP $\rightarrow$ pré-operatório. $P O \rightarrow$ pós-operatório 
Tabela 2

Triglicerídeos nos diferentes grupos e períodos operatórios

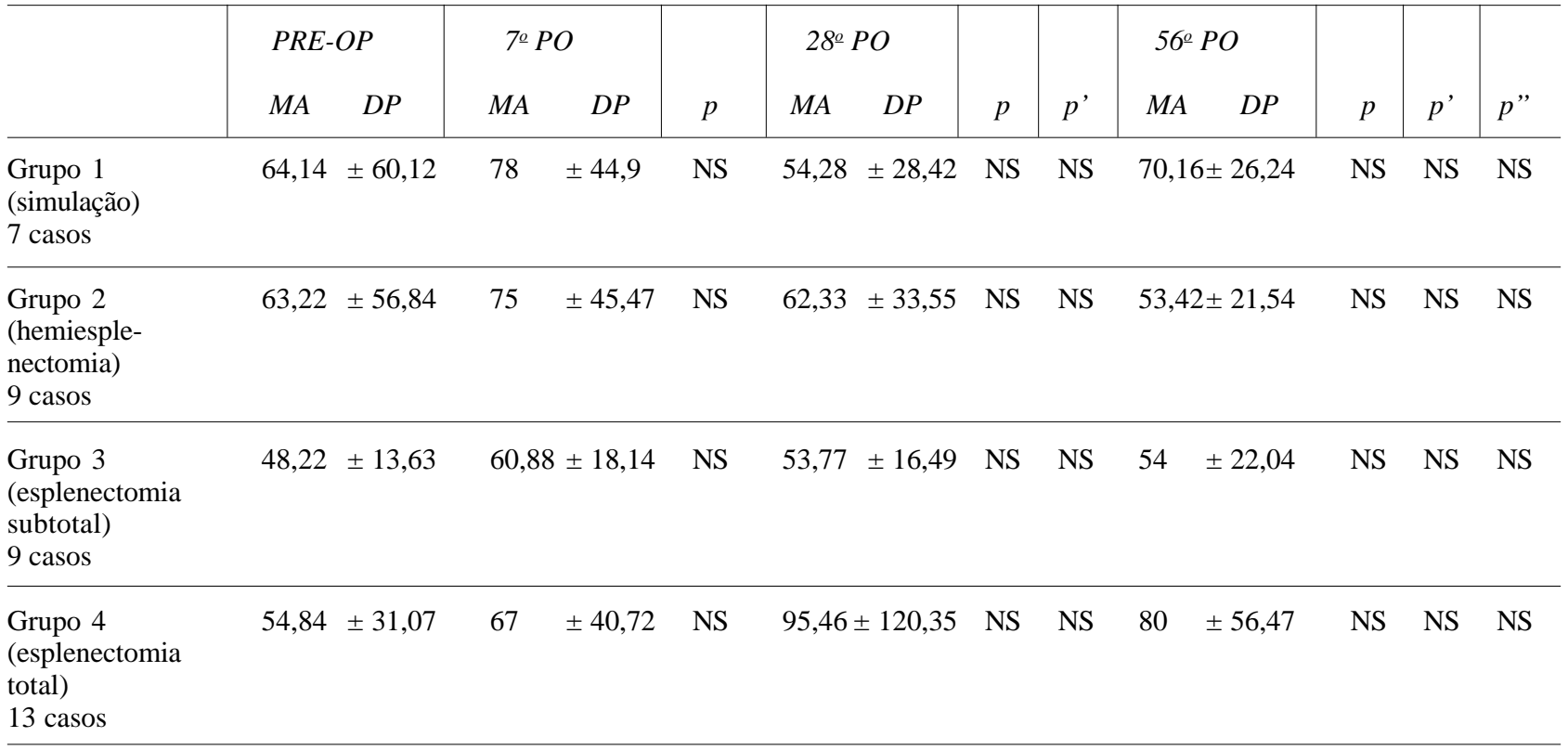

Análise de variância de medidas repetidas. Protected T test. $p$ - * $\rightarrow$ significante quando for menor que 0,05. $p * * \rightarrow$ significante quando for menor que 0,01 p-em relação ao pré-op; p'- em relação ao 7o P.O; p" em relação ao 28 o. NS- não significante. M.A- Média aritmética DP-Desvio-padrão. PRE-OP $\rightarrow$ pré-operatório. $P O \rightarrow$ pós-operatório.

Tabela 3

HDL nos diferentes grupos e períodos operatórios

\begin{tabular}{|c|c|c|c|c|c|c|c|c|c|c|c|c|c|c|}
\hline & \multicolumn{2}{|c|}{$P R E-O P$} & \multicolumn{2}{|c|}{$7^{\circ} \mathrm{PO}$} & \multirow[b]{2}{*}{$p$} & \multicolumn{2}{|c|}{$28^{o} P O$} & \multirow[b]{2}{*}{$p$} & \multirow[b]{2}{*}{$p^{\prime}$} & \multicolumn{2}{|c|}{$56^{o} P O$} & \multirow[b]{2}{*}{$p$} & \multirow[b]{2}{*}{$p^{\prime}$} & \multirow[b]{2}{*}{$p "$} \\
\hline & $M A$ & $D P$ & $M A$ & $D P$ & & $M A$ & $D P$ & & & $M A$ & $D P$ & & & \\
\hline $\begin{array}{l}\text { Grupo } 1 \\
\text { (simulado) } \\
7 \text { casos }\end{array}$ & 77,42 & $\pm 25,76$ & 87 & $\pm 29,29$ & NS & 93,14 & $\pm 39,17$ & NS & NS & 82,66 & $\pm 28,43$ & NS & NS & NS \\
\hline $\begin{array}{l}\text { Grupo } 2 \\
\text { (hemiesple- } \\
\text { nectomia) } \\
9 \text { casos }\end{array}$ & \multicolumn{2}{|c|}{$74,33 \pm 22,75$} & \multicolumn{2}{|c|}{$87,77 \pm 27,21$} & NS & 84,22 & $\pm 33,65$ & NS & NS & 78,85 & $\pm 16,24$ & NS & NS & NS \\
\hline $\begin{array}{l}\text { Grupo } 3 \\
\text { (esplenectomia } \\
\text { subtotal) } \\
9 \text { casos }\end{array}$ & 66 & $\pm 20,16$ & \multicolumn{2}{|c|}{$69,77 \pm 18,39$} & NS & 59,44 & $\pm 14,49$ & NS & NS & 68,66 & $\pm 15,09$ & NS & NS & NS \\
\hline $\begin{array}{l}\text { Grupo } 4 \\
\text { (esplenectomia } \\
\text { total) } \\
13 \text { casos }\end{array}$ & \multicolumn{2}{|c|}{$57,30 \pm 19,77$} & \multicolumn{2}{|c|}{$84,16 \pm 22,39$} & $* *$ & 74 & $\pm 16,98$ & $*$ & NS & 70,88 & $\pm 11,48$ & NS & NS & NS \\
\hline
\end{tabular}

Análise de variância de medidas repetidas. Protected T test. $\mathrm{p}-* \rightarrow$ significante quando for menor que 0,05 . $\mathrm{p}^{* *} \rightarrow$ significante quando for menor que 0,01 p- em relação ao pré-op; p'- em relação ao 7o PO; p" em relação ao 28‥ NS- não significante. MA- Média aritmética DP- Desvio-padrão. PRE-OP $\rightarrow$ pré-operatório. PO $\rightarrow$ pós-operatório.

\section{DISCUSSÃO}

Estudos clínicos e experimentais têm mostrado que o baço desempenha importante papel no metabolismo li- pídico $^{1,5}$. Sua retirada provoca alterações importantes no referido metabolismo. Isso tem sido atribuído à hiperfunção do SRE, distúrbio dos receptores de lipoproteínas no fígado, e à existência de um fator esplênico que pode cau- 
Tabela 4

LDL nos diferentes grupos e períodos operatórios

\begin{tabular}{|c|c|c|c|c|c|c|c|c|c|c|c|}
\hline & $P R E-O P$ & $7^{\circ} P O$ & & $28^{\circ} \mathrm{PO}$ & & & $56^{\circ} P C$ & & & & \\
\hline & $M A \quad D P$ & $M A \quad D P$ & $p$ & $M A \quad D P$ & $p$ & $p^{\prime}$ & $M A$ & $D P$ & $p$ & $p^{\prime}$ & $p "$ \\
\hline $\begin{array}{l}\text { Grupo } 1 \\
\text { (simulação) } \\
7 \text { casos }\end{array}$ & $38,02 \pm 23,60$ & $43,26 \pm 28,85$ & NS & $43,22 \pm 35,83$ & NS & NS & $61,06=$ & $\pm 32,06$ & NS & NS & NS \\
\hline $\begin{array}{l}\text { Grupo } 2 \\
\text { (hemiesple- } \\
\text { nectomia) } \\
9 \text { casos }\end{array}$ & $43,46 \pm 28,52$ & $70,66 \pm 80,32$ & NS & $47,42 \pm 34,35$ & NS & NS & $52,62=$ & $\pm 24,33$ & NS & NS & NS \\
\hline $\begin{array}{l}\text { Grupo } 3 \\
\text { (esplenectomia } \\
\text { subtotal) } \\
\text { casos }\end{array}$ & $73,33 \pm 22,73$ & $92,77 \pm 30,26$ & NS & $74,33 \pm 49,59$ & NS & NS & $65,77=$ & $\pm 41,05$ & NS & NS & NS \\
\hline $\begin{array}{l}\text { Grupo } 4 \\
\text { (esplenectomia } \\
\text { total) } \\
13 \text { casos }\end{array}$ & $45,06 \pm 15,47$ & $51,98 \pm 26,73$ & NS & $60,50 \pm 44,06$ & NS & NS & $113,77 \pm$ & $\pm 86,56$ & $* *$ & $* *$ & $* *$ \\
\hline
\end{tabular}

Análise de variância de medidas repetidas. Protected T test. $p$ - * $\rightarrow$ significante quando for menor que 0,05. $p * * \rightarrow$ significante quando for menor que 0,01 p-em relação ao pré-op; $p$ '- em relação ao 7o PO; $p$ ”em relação ao $28^{\circ}$. NS- não significante. MA-Média aritmética DP-Desvio-padrão. $P R E-O P \rightarrow$ pré-operatório. $P O \rightarrow$ pós-operatório.

Tabela 5

VLDL nos diferentes grupos e períodos operatórios

\begin{tabular}{|c|c|c|c|c|c|c|c|c|c|c|c|c|c|c|}
\hline & \multicolumn{2}{|c|}{$P R E-O P$} & \multicolumn{2}{|c|}{$7^{\circ} \mathrm{PO}$} & \multirow[b]{2}{*}{$p$} & \multicolumn{2}{|c|}{$28^{\circ} \mathrm{PO}$} & \multirow[b]{2}{*}{$p$} & \multirow[b]{2}{*}{$p^{\prime}$} & \multicolumn{2}{|c|}{$56^{o} \mathrm{PO}$} & \multirow[b]{2}{*}{$p$} & \multirow[b]{2}{*}{$p^{\prime}$} & \multirow[b]{2}{*}{$p "$} \\
\hline & $M A$ & $D P$ & $M A$ & $D P$ & & $M A$ & $D P$ & & & $M A$ & $D P$ & & & \\
\hline $\begin{array}{l}\text { Grupo } 1 \\
\text { (simulação) } \\
7 \text { casos }\end{array}$ & 12,82 & $\pm 12,02$ & 16,71 & $\pm 9,14$ & NS & 10,85 & $\pm 5,68$ & NS & NS & 14,03 & $\pm 5,24$ & NS & NS & NS \\
\hline $\begin{array}{l}\text { Grupo } 2 \\
\text { (hemiesple- } \\
\text { nectomia) } \\
9 \text { casos }\end{array}$ & 17,84 & $\pm 16,89$ & 15 & $\pm 9,09$ & NS & 12,46 & $\pm 6,71$ & NS & NS & 10,97 & $\pm 4,53$ & NS & NS & NS \\
\hline $\begin{array}{l}\text { Grupo } 3 \\
\text { (esplenectomia } \\
\text { subtotal) } \\
\text { casos }\end{array}$ & 9,64 & $\pm 2,72$ & 12,17 & $\pm 3,62$ & NS & 10,75 & $\pm 3,29$ & NS & NS & 10,8 & $\pm 4,40$ & NS & NS & NS \\
\hline $\begin{array}{l}\text { Grupo } 4 \\
\text { (esplenectomia } \\
\text { total) } \\
13 \text { casos }\end{array}$ & 13,56 & $\pm 9,38$ & 13,4 & $\pm 8,14$ & NS & $19,09=$ & $\pm 24,07$ & NS & NS & 16 & $\pm 11,29$ & NS & NS & NS \\
\hline
\end{tabular}

Análise de variância de medidas repetidas. Protected T test. $p$ - * $\rightarrow$ significante quando for menor que 0,05. $p * * \rightarrow$ significante quando for menor que 0,01 p-em relação ao pré-op; p'- em relação ao 7o PO; p" em relação ao 28 o. NS- não significante. MA- Média aritmética DP-Desvio-padrão. PRE-OP $\rightarrow$ pré-operatório. $P O \rightarrow$ pós-operatório.

sar hipocolesterolemia no hiperesplenismo ou suprimir a hiperlipidemia ${ }^{6}$. Nosso trabalho mostrou que no grupo com operação simulada não houve alteração significante do li- pidograma. O grupo da esplenectomia total mostrou variações importantes do colesterol total, HDL e VLDL. Isso mostra que o baço interfere no metabolismo lipídico. 
É importante lembrar que o HDL aumentou significantemente no 7o e no $28^{\circ} \mathrm{PO}$ em relação ao pré-operatório, e que o LDL aumentou significantemente no 56을, em relação ao pré-operatório. Considerando-se o LDL uma fração aterogênica, é de se supor que a esplenectomia total pode predispor à aterogênese.

O grupo de animais submetido à hemiesplenectomia não mostrou alterações significantes no lipidograma. Houve apenas aumento significante do colesterol no sétimo PO em relação ao pré-operatório. Isso mostra que cerca de $42 \%$ do baço na fase inicial não são capazes de equilibrar o metabolismo lipídico, embora os valores das frações do colesterol não tenham sofrido modificações importantes. Esse equilíbrio, no entanto, pode ser verificado no $28^{\circ}$ e no 56을. Nos animais submetidos à esplenectomia subtotal, nos quais realizamos a ligadura do tronco principal da artéria e da veia esplênicas $^{8}$, não houve aumento significante do colesterol entre os diferentes períodos operatórios. Esse fato mostra que o remanescente esplênico, mesmo sem um ramo do tronco principal dos vasos esplênicos, mantém a influência do baço no metabolismo lipídico. Isso é curioso, pois o peso do baço deixado na esplenectomia subtotal é menor do que aquele deixado na hemiesplenectomia onde ocorreram alterações significantes no 7ํ $\mathrm{PO}$ em relação ao pré-operatório. Convém lembrar que não houve diferença entre os pesos dos dois grupos ( $\mathrm{p}>0,05)$. É lógico, portanto, admitir que um fator desconhecido interferiu no sétimo PO do grupo da hemiesplenectomia, trazendo resultados inesperados no nível de colesterol total. Cumpre ressaltar que todos os animais estavam se alimentando de uma dieta padrão, mantidos no mesmo ambiente e nas mesmas condições. É importante assinalar também que a remoção do órgão produziu aumento significante do colesterol, LDL e HDL. Resultados diferentes foram observados em coelhos submetidos à esplenectomia. Nesses animais verificou-se que a esplenectomia produziu aumento do colesterol e triglicerídeos e diminuição do $\mathrm{HDL}^{6}$. Em ratos observou-se aumento significante dos triglicerídeos e diminuição do HDL. ${ }^{7}$ Em cães, os efeitos da esplenectomia sobre o metabolismo lipídico são controver$\operatorname{tidos}^{4,5}$. Em nossa investigação ficou claro que a remoção dessa víscera produz alterações no metabolismo lipídico. Outras questões poderiam ser levantadas a partir dessa investigação: $1^{\mathrm{a}}$ - O que aconteceria no metabolismo lipídico num grupo de animais submetidos a implante de baço? $2^{\mathrm{a}}-$ O pólo superior do baço suprido pelos vasos gastroesplênicos seria capaz de manter também a função lipídica? 3a - $\mathrm{O}$ que ocorreria com o metabolismo lipídico dos animais submetidos à esplenectomia total e hemiesplenectomia a longo prazo tendo um grupo simulação como controle?; 4a - $\mathrm{O}$ que ocorreria em animais esplenectomizados que se alimentassem de diferentes tipos de dietas, que poderiam interferir nos níveis lipídicos? 5a — Do mesmo modo, o que aconteceria em animais esplenectomizados que usassem medicamentos que teriam também interferência nos níveis de colesterol e frações? 6 ${ }^{\mathrm{a}}$ — Confirmando-se que os pólos esplênicos têm vascularização diferente, especialmente a arterial, justifica-se também o estudo comparativo desses dois grupos. Essas e outras questões poderiam ser levantadas. Mas o que importa é que a esplenectomia induziu alterações lipídicas importantes, e que a hemiesplenectomia e a esplenectomia subtotal com preservação do pólo inferior, não.

Do que foi exposto, julgamos poder concluir:

- O baço interfere no metabolismo lipídico. Houve aumento significante do colesterol total, HDL e LDL no pós-operatório dos cães submetidos à esplenectomia total. No 56을 PO houve aumento significante do LDL e não-significante do HDL

- A conservação da metade inferior do baço foi capaz de evitar alterações no metabolismo lipídico no $28^{\circ}$ e $56^{\circ}$ dias pós-operatórios. A conservação do pólo inferior do baço foi também capaz de evitar alterações significantes no lipidograma em todo o período pós-operatório.

\begin{abstract}
Background: The purpose of this study was to evaluate the effect of partial (hemi-splenectomy and subtotal splenectomy) and total splenectomy in the dogs lipidic profile. Method: Thirty eight (38) mongrel male dogs, with weight varying between $13 \mathrm{Kg}$ and $15 \mathrm{Kg}$ were operated. After clinical evaluation and twelve hour fasting of standard diet, preoperatively hemogram and lipidic profile were evaluated. After general anaesthesia with sodium thiopental, the dogs were submitted to an upper and lower median laparotomy. Group I, seven dogs, just manipulation of the spleen (sham-control); group II, nine dogs upper hemisplenectomy; group III, nine dogs, subtotal splenectomy with preservation of inferior pole, after the ligature and section of the splenic artery and vein near the hilus; group IV, thirteen dogs, total splenectomy was performed. Results: Our results, when we compared to the lipid values of the different post-operative periods with preoperative data, showed that in group IV (total splenectomy) there was a significant increase of the total cholesterol in all post-operative periods $(p<0,05, p<0,01)$. The high-density lipoprotein $(H D L)$ was significantly augmented in the $7^{\text {th }}(p<0,01)$ and $28^{\text {th }}(p<0,05)$ post-operative day and at not significantly $(p>0,05)$ on the $56^{\text {th }}$ post-operative time. The low-density lipoprotein $(L D L)$ increased significantly in the $56^{\text {th }}$ post-operative $(p<0,01)$. Triglyceride and very-low density lipoproteins $(V L D L)$ did not present significant changes. Animals of group 1 and 3 did not present significant changes. In group 2 there was a significant increased in cholesterol on the $7^{\text {th }}$ post-operative day. There was no difference between the weight of the spleen remaining after partial splenectomy. Conclusions: We conclude that total splenectomy causes significant elevation in the total cholesterol, HDL and LDL, while triglycerides and VLDL don't change; - the preservation of the inferior lower half of the spleen and the preservation of the inferior pole on subtotal splenectomy protected the animal of significant changes in lipidic profile.
\end{abstract}

Key Words. Partial splenectomy; Total splenectomy; Splenectomy and Cholesterol. 


\section{REFERÊNCIAS}

1. Aviram M, Brook JG, Tatarsky I et al. Increased low-density lipoprotein levels after splenectomy: A role for the spleen in cholesterol metabolism in myeloproliferative disorders. Am J Med Sci 1986; 291: 25-28.

2. Sugihara T, Yawata Y. Observations on plasma and red cell lipids in hereditary spherocytosis. Clin Chim Acta 1984; 137: 227-32.

3. Robinete CD, Fraumeni JF Jr. Splenectomy and subsequent mortality in veterans of the 1939-45 war. Lancet 1977; 16: 127-9.

4. King JH. Studies in the pathology of the spleen. Arch Intern Med 1914; 14: 145-167.

5. Ham JM, Furneaux RW. The effect of splenectomy on blood platelets and lipoprotein lipase activity in the dog. Brit J Surg 1969; 56: 527-30.

6. Asai K, Kuzuya M, Naito M et al. Effects of splenectomy on serum lipids and experimental atherosclerosis. Angiology 1988; 39: 497-504.
7. Fatouros M, Bourantas K, Bairaktari E et al. Role of the spleen in lipid metabolism. Br J Surg 1995; 82: 16751677.

8. Paulo DNS, Lázaro da Silva A, Cintra LC et al. Esplenectomia subtotal, em cães, com preservação do pólo inferior suprido por vasos do ligamento gastroesplênico. Rev Col Bras Cir 1999; 26: 147-52.

Endereço para Correspondência do 1ํA Autor Rua Santa Rita de Cássia, 439//101 29042-260 — Vitória-ES 